\title{
Using the Constraint Language Toupie for "Software Cost Reduction" Specification Analysis
}

\author{
Antoine Rauzy*
}

\begin{abstract}
Constraint programming has attracted a considerable interest over recent years. It has been successfully applied to combinatorial optimization problems that were up to now considered as difficult. In this presentation, the constraint language Toupie is used to analyze the specification of a reactive system formulated in terms of the "Software Cost Reduction" method. Our aim is to show that the constraint programming paradigm is relevant for modelization and verification of embedded systems as well.
\end{abstract}

* LaBRI - CNRS URA 1304 - Université Bordeaux 51, cours de la Libération, F-33405 Talence (France), email: rauzyelabri.u-bordeaux.fr . 